\title{
Disseminated Abscessation Complicated with Bone Marrow Abscess Caused by Arcanobacterium pyogenes in a Goat
}

\author{
Cheng-Chung LIN ${ }^{1)}$, Ter-Hsin CHEN ${ }^{1}$, Ching-Lin SHYU' ${ }^{2)}$, Nan-Yu SU³ ${ }^{3)}$ and Jacky Peng-Wen CHAN ${ }^{2) *}$ \\ 1) Graduate Institute of Veterinary Pathobiology, College of Veterinary Medicine, National Chung Hsing University, 250 Kuo-Kuang Rd., \\ Taichung 40227 and ${ }^{2}$ Department of Veterinary Medicine and Veterinary Medical Teaching Hospital, College of Veterinary Medicine, \\ National Chung Hsing University, 250 Kuo-Kuang Rd., Taichung 40227, Taiwan
}

(Received 1 December 2009/Accepted 17 March 2010/Published online in J-STAGE 12 May 2010)

ABSTRACT. An 8-month-old, Nubian wether with a history of systemic illness was euthanatized for a pathological examination. At necropsy, the presence of disseminated abscessation and cellulitis in the limbs was noted. Other postmortem findings associated with the visceral disease in this animal included multiple abscess lesions, mainly in the lungs, kidneys, phalanxes and vertebrae. Histopathologically, lesions of arteriolitis were found as evidenced by bacterial embolisms in pulmonary and renal arteriola, indicating a bacteremia in the patient. Arcanobacterium pyogenes was consistently isolated from 8 lesions of abscessations, including the lesions of subcutaneous abscesses as well as bone marrow abscess in phalanxes and thoracic vertebrae. This is the first published report of disseminated arcanobacterial infection with bone marrow abscess of both the phalanxes and vertebrae in goat.

KEY WORDS: abscessation, Arcanobacterium pyogenes, bone marrow abscess, goat.

J. Vet. Med. Sci. 72(8): 1089-1092, 2010

Arcanobacterium pyogenes (formerly named Actinomyces pyogenes and Corynebacterium pyogenes) [19] is associated with production of abscesses and pus, particulary in ruminants $[10,16]$. This organism is one of the several bacterial species that constitute the common resident on the mucosa of urogenital tract, upper respiratory tract and ruminal wall in ruminants and swine [4, 7]. A. pyogenes is also an opportunistic pathogen which may survive in humid environment for up to 10 months [16] and can disseminate to cause suppurative infections involving the skin, joints, lymph nodes and feet $[4,7,10,16]$ as well as a variety of visceral lesions such as liver abscess, kidney abscess, intracranial abscess, purulent pneumonia, osteomyelitis, and pyometra in several animal species $[7,10,15,17,20,21]$. Corynebacteria spp. have been isolated from the udder infected with mastitis in ewes, whereas $10 \%(5 / 50)$ of them were recognized as $A$. pyogenes [8]. The present report describes the clinical presentation and pathological findings in a Nubian goat with disseminated abscessation complicated with bone marrow abscess due to $A$. pyogenes infection.

The herd was comprised of approximately 200 Nubian goats of varying ages. All the goats were maintained under uniform feeding and were generally in good body condition all year round. The animals were group-housed under intensive system of rearing confined in several identical pens (3 $\times 3 \mathrm{~m}$ ) with slatted floor. On average, 15 goats were raised in a pen. The owner reported that 5 goats had progressive signs of multiple fluctuating swellings on limbs, which had gradually enlarged over a period of 3-4 weeks. As none of

\footnotetext{
* Correspondence to: Chan, J. P.-W., Department of Veterinary Medicine, College of Veterinary Medicine, National Chung Hsing University, 250 Kuo-Kuang Rd., Taichung 40227, Taiwan.

e-mail: pwchan@dragon.nchu.edu.tw
}

the 5 goats responded positively to daily treatment with lincomycin and corticosteriod, also presenting recumbency with abnormal general condition, 4 of them were culled. The other, an 8-month-old, $37 \mathrm{~kg}$, Nubian wether was submitted to the hospital for evaluation of this disease.

On presentation, the goat was recumbent and showed signs of dullness with pyrexia up to $41.1^{\circ} \mathrm{C}$, multiple abscess lesions under the skin of the limbs. Over-growing and cracks of the hooves were noted. Abnormal findings during physical examination included an estimated dehydration of $3-5 \%$, absence of rumen motility and prolonged capillary refill time $(>3 \mathrm{sec})$. There was no evidence of wounds on the body.

Blood abnormalities included severe macrocytic, hypochromic anemia with marked thrombocytopenia, leucocytosis $(20,100$ leucocytel $/ \mu l)$ and an absolute neutrophilia $(8,844$ neutrophils/ $\mu l)$ with a moderate left shift (bands $1,005$ cells $/ \mu l)$. There were markedly increased activities of aspartate aminotransferase (166.6 U/l), lactate dehydrogenase $(527 \mathrm{U} / l)$, alkaline phosphatase $(151 \mathrm{U} / l)$, and creatinine kinase $(90 \mathrm{U} / l)$. The levels of albumin $(2.0 \mathrm{~g} / \mathrm{d} l)$ and $\mathrm{A} /$ $\mathrm{G}$ ratio $(0.42)$ were lower than normal, as were the concentrations of serum calcium $(7.0 \mathrm{mg} / \mathrm{d} l)$ and magnesium $(0.8$ $\mathrm{mg} / \mathrm{d} l$ ). The differential diagnosis included caseous lymphadenitis, bacterial polyarthritis and Mycoplasma arthritis. Medical intervention was not an option and the goat was euthanatized for a pathological examination with the owner's consent.

Postmortem examination revealed the presence of cellulitis extending from carpus to shoulder of the right forelimb as well as in the area of metatarsus of the right hindlimb, which was completely filled with large amounts of whitish-yellow purulent exudates between the epidermis and muscularis. Multiple swellings as a result of pus accumulation in the area of the shoulder on the left forelimb were seen, in which 


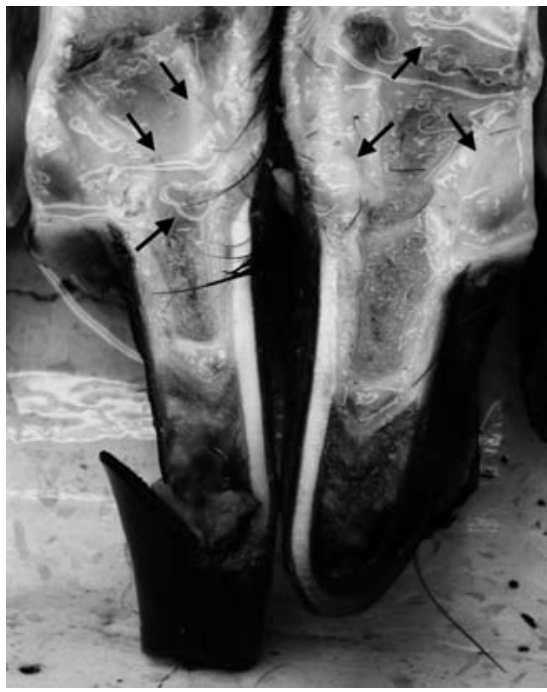

Fig. 1. Sagittal section through the overgrowing hoof on right forelimb showing sole ulceration and osteomyelitis in $2^{\text {nd }}$ and $3^{\text {rd }}$ phalanxes, in which purulent exudate (arrows) was noted.

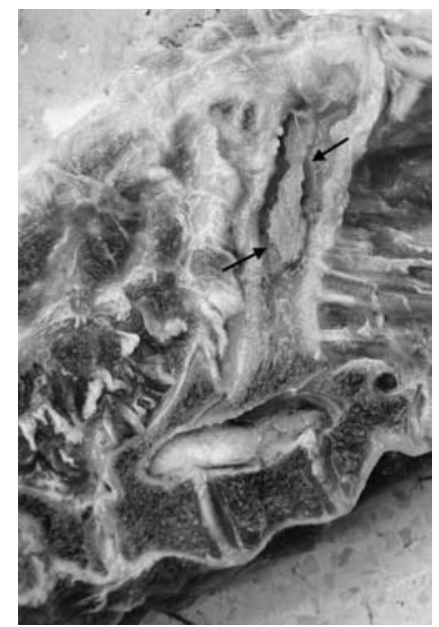

Fig. 2. Sagittal section of the $4^{\text {th }}$ thoracic vertebra of the wether showing an abscess (arrows) in the spinous process.

the arthrodial cartilage was invaded by evidence of fibrinous adhesion. Totally, 4.8 liters of pus was drained from the cellulitis and abscess lesions. The feet showed severe sole ulceration with related bone marrow abscess in phalanxes (Fig. 1). Other postmortem findings associated with the visceral disease in this animal included multiple abscess lesions, mainly in the lungs (pleurobronchopneumonia), kidneys and vertebrae starting from the 4th cervical vertebra to the 4 th thoracic vertebra (Fig. 2). The lymph nodes involved in the areas of abscessations were grossly

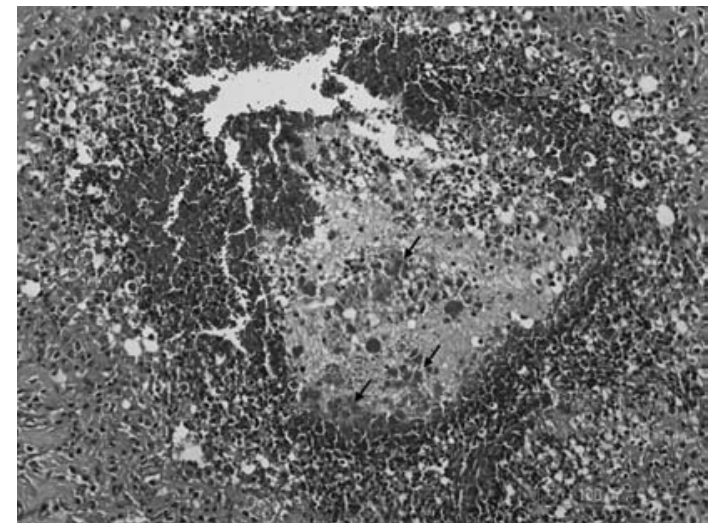

Fig. 3. Lung. A lesion of thrombus was evident in pulmonary arteriole, predominantly packed with neutrophils, fibrinoid material and bacterial clumps (arrows) in the lumen, while arteriolar wall was infiltrated with neutrophils. HE. Bar $=100 \mu \mathrm{m}$.

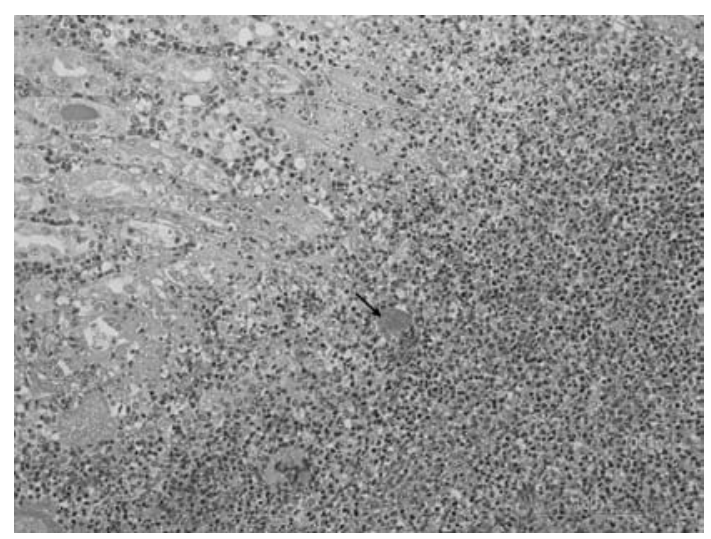

Fig. 4. Kidney. Margin of the infarct in the cortex was filled with neutrophils, cellular debris, and bacterial clumps (arrow). HE. Bar $=100 \mu \mathrm{m}$.

enlarged. At histopatholgical examination, lesions of arteriolitis packed with thrombi and scattered bacterial clumps were noted throughout the lung (Fig. 3). Mild congestion and emphysema were seen in the pulmonary parenchyma, while widespread lesions of necrotizing bronchitis were evident by large amount of bacterial clumps and fibrinoid material. Multifocal wedge shaped infarcts were mostly found at the end of the thrombolized arcuate arteries. Additionally, multiple infarctions were present in the cortex of the kidney (Fig. 4). Well demarcated necrotic renal infarctions were filled with neutrophils, cellular debris, and bacterial clumps. Arterial thrombosis was found as evidenced by bacterial embolisms in pulmonary and renal arteriola which were predominantly infiltrated with numerous neutrophils in the arteriolar walls. Gram-stained impression smears from the bone marrow revealed numerous rod shaped Grampositive bacteria, occurring single or in clumps, mixed with neutrophils and necrotic trabecula indicating bone marrow 
abscess in the vertebra and phalanxes.

Swab specimens for bacterial culture were obtained from 8 lesions of absessations, including the lesions of subcutaneous abscesses and bone marrows in phalanxes and thoracic vertebrae. All the swabs specimens yielded a pure, heavy growth of $A$. pyogenes, which was sensitive to cephalothin, flofenicol and trimethroprim/sulfamethoxazole according to related disc agar diffusion antimicrobial susceptibility test by Clinical and Laboratory Standards Institute (CLSI). The results of bacterial culture were consistent with the findings of Gram-stained smear from the swabs and the bone marrow smears, showing clusters of Gram-positive rods. However, there was a paucity of histopathological evidence of the bone specimen and no organism was isolated from the blood sample. Based on the pathological and microbiological findings, a case of disseminated abscessation complicated with bone marrow abscess due to $A$. pyogenes infection was diagnosed.

A variety of organisms causing subcutaneous abscess have been isolated in goats, such as Corynebacterium pseudotuberculosis, A. pyogenes, Escherichia coli, and Staphylococcal and Streptococcal species [24]. The organisms may enter the blood stream and cause bacteremia, abscesses and suppurative lesions in various tissue and organs [7]. Abscessation results from infection with $A$. pyogenes, which usually gains entry through puncture wounds or abrasions of the skin, showing lesions particularly on the head and body $[10,24]$. However, no wound was found on the body of this wether, except the sole ulceration in hooves. Foot abscessation may occur as a result of invasion of Fusobacterium necrophorum, which, along with $A$. pyogenes, is usually responsible for hoof infection in small ruminant animals $[16,24,25]$, however, $F$. necrophorum from the hoof lesions of this wether was not isolated.

Pyogenic infection and abscess formation have been previously reported in sheep which affected meat quality and caused the death of the suffering animals, in which, A. pyogenes infection had a prevalence of $5.9 \%$ in this sheep flock [5]. In this case, because the purulent exudates accumulated in the deep subcutaneous tissue and muscle, the spread of abscesses contributed to contamination of goats meat, causing the threat to the public hygiene and food safety. Culling of affected goats was suggested. It is suggested that prompt medical intervention at the early stage of abscess formation is necessary to bring about a favourable outcome [24].

Bacterial infection of the bones is rare in goats [10, 24]. A previous report has described a case of osteomyelitis of the ribs associated with Corynebacterium renale in a goat [1]. In the present case, pathological examinations revealed enlargement of the regional lymph nodes as well as bacterial embolisms in pulmonary and renal arteriola, indicating a bacteremia in the patient. The pathological evidences suggested that both lesions of bone marrow abscess in vertebrae and phalanxes occurred secondary to haematogenous spread from disseminated abscessation, although no organism was isolated from the blood sample.

Since we can only show the bacteriological evidence of the bone marrow smear, it could be speculated reasonably, that osteomyelitis was the case in the phalanxes and thoracic vertebrae. Osteomyelitis is believed to generally result from the hematogeneous spread of a remote focus of infection or post-traumatic contamination of microbial seeding of the bone in humans, cattle and horses [9, 11, 13, 22, 27]. Staphylococcus aureus is the most prevalent causative microorganism of hematogenous osteomyelitis in humans [13, 22], which occurs predominately in children $[6,14,22]$. In the majority of cases, vertebral osteomyelitis is hematogenous in origin $[2,22]$, sources of bacteremia include a trivial skin infection, infective endocarditis, or a serious infection of the respiratory, gastrointestinal or genitourinary tracts [14, 22]. In cattle, salmonella and Corynebacterium pyogenes were mostly isolated from the bone lesions of epiphyseal and physeal osteomyelitis, respectively [9]. In horses, enterobacteriaceae are most commonly isolated in cases of hematogenous osteomyelitis in foals as a result of sepsis, which occurs mostly in a joint, epiphysis, or physis [11]. Additionally, cases of mycotic osteomyelitis presenting lesions in the proximal phalanx, rib and metacarpophalangeal joint, have also been reported in young horses [18, 26].

The bacterial colonization in the bone is required in order to initiate infection [12]. Bacteria can adhere to tissues by binding host proteins, including fibrinogen, fibronectin and collagen [6]. For instance, collagen-binding adhesin (Cna) makes an important contribution to the ability of $S$. aureus to bind collagen, which is encoded by a gene (Cna) of $S$. aureus, but not present in most strains $[6,23]$. This function leads to the bacterial attachment and formation of biofilm with the subsequent growth of sessile bacteria on the host tissue $[11,12,23]$. However, it is not known whether $A$. pyogenes is consistent with the two-stage process described above to establish infection in bone.

It has been reported that $A$. pyogenes isolated from cattle and swine was fully sensitive to benzylpenicillin and ampicillin, moderately sensitive to ceftiofur, cefapirin, and resistant to dihydrostreptomycin and oxytetracycline [29]. A similar result has been found by Trinh et al. [28], where $70.6 \%$ of porcine, but only $25.9 \%$ of bovine $A$. pyogenes isolates were resistant to tetracyclines. Our result indicated that $A$. pyogenes isolate was resistant to penicillin, oxytetracycline, ceftiofur, enrofloxacin, and lincomycin, and was sensitive to cephalothin, flofenicol and trimethroprim/sulfamethoxazole. Frequent use of antibiotics may lead to the emergence of resistant $A$. pyogene strains and additionally the potential for antibiotic residues in meat.

We speculate that the sporadic outbreak as a result of high rearing density in this flock leading to intimate contact among goats, which predisposed the animals to the $A$. pyogenes infection. Such a stress factor probably affected the animal's resistance, contributing to the spread of the pathogen to subcutaneous tissues and thence to other viscera. Control measures in this herd might include decreasing overall rearing density, hoof care and trimming, removing any sharp pointed objects in the barn, and maintaining goats 
in clean and dry areas. The case was followed up by culling 2 goats suffering from subcutaneous abscesses and mastitis caused by $A$. pyogenes, respectively. The disease outbreak has been completely controlled at the following farm visiting.

Although the pathological lesions of this case were in line with what has been previously described in ruminants $[3,5$, $7,16]$, the authors believe that this is the first published report of disseminated arcanobacterial infection with bone marrow abscess of both the phalanxes and vertebrae in goat.

ACKNOWLEDGMENT(S). The authors would like to acknowledge the assistance of Miss A. Jack for the English revision.

\section{REFERENCES}

1. Altmaier, K. R., Sherman, D. M., Schelling, S. H., Fister, R. D. and Lamb, C. R. 1994. Osteomyelitis and disseminated infection caused by Corynebacterium renale in a goat. J. Am. Vet. Med. Assoc. 204: 934-937.

2. Barnea, Y., Carmeli, Y., Kuzmenko, B. and Navon-Venezia, S. 2007. Staphylococcus aureus mediastinitis and sterna osteomyelitis following median sternotomy in a rate model. J. Antimicrob. Chemother. 62: 1339-1343.

3. Bath, G. F., van Wyk, J. A. and Pettey, K. P. 2005. Control measures for some important and unusual goat diseases in southern Africa. Small Ruminant Res. 60: 127-140.

4. Billington, S. J., Post, K. W. and Jost, B. H. 2002. Isolation of Arcanobacterium (Actinomyces) pyogenes from cases of feline otitis externa and canine cystitis. J. Vet. Diagn. Invest. 14: 159-162.

5. Dughaym, A. M. A. 2004. Isolation of Serratia, Arcanobacterium and Burkholderia species from visceral and cutaneous abscesses in four emaciated ewes. Vet. Rec. 155: 425-426.

6. Elasri, M. O., Thomas, J. R., Skinner, R. A., Blevins, J. S., Beenken, K. E., Nelson, C. L. and Smeltzer, M. S. 2002. Staphylococcus aureus collagen adhesin contributes to the pathogenesis of osteomyelitis. Bone 30: 275-280.

7. Ertas, H. B., Kilic, A., Ozbey, G. and Muz, A. 2005. Isolation of Arcanobacterium (Actinomyces) pyogenes from abscessed cattle kidney and identification by PCR. Turk. J. Vet. Anim. Sci. 29: 455-459.

8. Fernández, E. P., Vela, A. I., Las-Heras, A., Domínguez, L., Fernández-Garayzábal, J. F. and Moreno, M. A. 2001. Antimicrobial susceptibility of Corynebacteria isolated from ewe's mastitis. Int. J. Antimicrob. Agents 18: 571-574.

9. Firth, E. C., Kersjes, A. W., Dik, K. J. and Hagens, F. M. 1987. Haematogenous osteomyelitis in cattle. Vet. Rec. 120: 148152.

10. Gezon, H. M., Bither, H. D., Hanson, L. A. and Thompson, J. K. 1991. Epizootic of external and internal abscesses in a large goat herd over a 16-year period. J. Am. Vet. Med. Assoc. 198: 257-263.

11. Goodrich, L. R. 2006. Osteomyelitis in horses. Vet. Clin. N. Am. Equine Pract. 22: 389-417.

12. Gristina, A. G., Oga, M., Webb, L. X. and Hobgood, C. D. 1985. Adherent bacterial colonization in the pathogenesis of osteomyelitis. Science 228: 990-993.
13. Harris, L. G. and Richards, R. G. 2006. Staphylococci and implant surface: a review. Injury 37: 3-14.

14. Kara, A., Tezer, H., Devrim, I., Caglar, M., Cengiz, A. B., Gür, D. and Secmeer, G. 2007. Primary sternal osteomyelitis in a healthy child due to community-acquired methicillin-resistant Staphylococcus aureus and literature review. Scand. J. Infect. Dis. 39: 469-472.

15. Karns, G. R., Lancia, R. A., DePerno, C. S., Conner, M. C. and Stoskopf, M. K. 2009. Intracranial abscessation as a natural mortality factor for adult male white-tailed deer (Odocoileus virginianus) in Kent County, Maryland, USA. J. Wildl. Dis. 45: 196-200.

16. Lavín, S., Ruiz-Bascarán, M., Marco, I., Abarca, M. L., Crespo, M. J. and Franch, J. 2004. Foot infections associated with Arcanobacterium pyogenes in free-living fallow deer (Dama dama). J. Wildl. Dis. 40: 607-611.

17. Lechtenberg, K. F., Nagaraja, T. G., Leipold, H. W. and Chengappa, M. M. 1988. Bacteriologic and histologic studies of hepatic abscesses in cattle. Am. J. Vet. Res. 49: 58-62.

18. Lenard, Z. M., Lester, N. V., O'hara, A. J., Hopper, B. J. and Lester, G. D. 2007. Disseminated cryptococcosis including osteomyelitis in a horse. Aust. Vet. J. 85: 51-55.

19. Quinn, P. J., Markey, B. K., Carter, M. E., Donnelly, W. J. and Leonard, F. C. 2002. Actinomycetes. pp. 63-71. In: Veterinary Microbiology and Microbial Disease, Blackwell Science, London.

20. Seifi, H. A., Saifzadeh, S., Farshid, A. A., Rad, M. and Farrokhi, F. 2003. Mandibular pyogranulomatous osteomyelitis in a sanen goat. J. Vet. Med. Ser. A. 50: 219-221.

21. Semambo, D. K. N., Ayliffe, T. R., Boyd, J. S. and Taylor, D. J. 1991. Early abortion in cattle induced by experimental intrauterine infection with pure cultures of Actinomyces pyogenes. Vet. Rec. 129: 12-16.

22. Sia, I. G. and Berbari, E. F. 2006. Osteomyelitis. Best Pract. Res. Clin. Rheumatol. 20: 1065-1081.

23. Smeltzer, M. S. and Gillaspy, A. F. 2000. Molecular pathogenesis of staphylococcal osteomyelitis. Poult. Sci. 79: 10421049.

24. Smith, M. C. and Sherman, D. M. 1994. Musculoskeletal system. pp. 63-121. In: Goat Medicine, 1st ed. (Smith, M. C. and Sherman, D. M. eds), Williams and Wilkins, Baltimore.

25. Stover, S. M. 2002. Disease of the bones, joints and connective tissue. pp. 1085-1131. In: Large Animal Internal Medicine, 3rd ed. (Smith, B. P. ed), Mosby, St. Louis.

26. Swerczek, T. W., Donahue, J. M. and Hunt, R. J. 2001. Scedosporium prolificans infection associated with arthritis and osteomyelitis in a horse. J. Am. Vet. Med. Assoc. 218: 1800 1802.

27. Swinebroad, E. L., Dabareiner, R. M., Swor, T. M., Carter, G. K., Watkins, J. P., Walker, M., Schmitz, D. G. and Honnas, C. M. 2003. Osteomyelitis secondary to trauma involving the proximal end of the radius in horses: five cases (1987-2001). $J$. Am. Vet. Med. Assoc. 223: 486-491.

28. Trinh, H. T., Billington, S. J., Field, A. C., Songer, J. G. and Jost, B. H. 2002. Susceptibility of Arcanobacterium pyogenes from different sources to tetracycline, macrolide and lincosamide antimicrobial agents. Vet. Microbiol. 85: 353-359.

29. Yoshimura, H., Kojima, A. and Ishimaru, M. 2000. Antimicrobial susceptibility of Arcanobacterium pyogenes isolated from cattle and pigs. J. Vet. Med. Ser. B. 47: 139-143. 